The University of San Francisco

USF Scholarship: a digital repository @ Gleeson Library | Geschke Center

Nursing and Health Professions Faculty Research and Publications

School of Nursing and Health Professions

2007

\title{
Behavioral Management of Command Hallucinations to Harm in Schizophrenia
}

Robin Buccheri

University of San Francisco, buccherir@usfca.edu

L Trygstad

G Dowling

Follow this and additional works at: http://repository.usfca.edu/nursing_fac

Part of the Nursing Commons, and the Psychiatric and Mental Health Commons

\section{Recommended Citation}

Buccheri R., Trygstad L., Dowling G.(2007). Behavioral Management of Command Hallucinations to Harm in Schizophrenia. J Psychosoc Nurs Ment Health Serv. 45(9) 46-54.

This Article is brought to you for free and open access by the School of Nursing and Health Professions at USF Scholarship: a digital repository @ Gleeson Library | Geschke Center. It has been accepted for inclusion in Nursing and Health Professions Faculty Research and Publications by an authorized administrator of USF Scholarship: a digital repository@ Gleeson Library | Geschke Center. For more information, please contact repository@usfca.edu. 


\section{Behavioral Management of Command Hallucinations in Schizophrenia}

When patients experience auditory hallucinations, it is critical to determine if their voices are commanding them to harm themselves or others. Such voices are often very distressing and some people are unable to resist complying with the commands (Mackinnon, Copolov, \& Trauer, 2004). Recently a 23-year old woman with schizophrenia who had stopped taking her medications followed her harm command hallucinations and threw her three young sons into the San Francisco Bay (AP, 2005).

\section{Purpose}

Our previous research in people with schizophrenia who experience persistent auditory hallucinations demonstrated that attending a 10-session course that taught behavioral strategies for managing auditory hallucinations was related to significant clinical improvements (i.e., frequency, self-control, clarity, tone, distractibility, and distress of auditory hallucinations, anxiety and depression) (Buccheri et al, 2004; Buccheri, Trygstad, Kanas, \& Dowling, 1997; Buccheri, Trygstad, Kanas, Waldren, \& Dowling, 1996; Trygstad, et al, 2002).

The purpose of this paper is to report findings related to command hallucinations to harm self or others. The specific aims are to describe: a) prevalence; b) demographic and clinical characteristics; c) changes in prevalence, characteristics and intensity of auditory hallucinations, and levels of anxiety and depression immediately after attending the course and at one-year post course; d) perceived helpfulness of the course; e) effectiveness of the 10 strategies taught in the course; and f) how individual data differ from grouped data in participants who report hearing command hallucinations to harm self or others.

\section{Review of the Literature}

Prevalence and compliance rates for command hallucinations 
Command hallucinations are relatively common among psychiatric patients with reports of prevalence rates among adult psychiatric patients ranging from $18-89 \%$ with a median of $53 \%$. Prevalence rates for "dangerous or harmful" command hallucinations range from 7-70\% with a median of 48\% (Shawyer, Mackinnon, Farnall, Tauer, \& Copolov, 2003).

Compliance with command hallucinations is a serious social concern. While reports are variable, one study of hospitalized psychiatric patients with psychoses found the majority of those who heard command hallucinations had complied with them during the past month. Specifically, $90 \%$ of patients complied with harmless commands, $92 \%$ with commands to harm self, and $67 \%$ with commands to harm others, resulting in an overall compliance rate of $84 \%$ (Kasper, Rogers, \& Adams, 1996).

\section{Predicting compliance with harm command hallucinations}

Eleven controlled studies have examined the relationships between hearing harm command hallucinations (i.e., violent and/ or suicidal behavior) and compliance with the commands (Rudnick, 1999). Four studies found direct relationships between compliance with harm commands and two particular characteristics of command hallucinations: voice familiarity/knowing the identity of the voice (Junginger, 1990, 1995) and voice benevolence (Beck-Sander, Birchwood, \& Chadwick, 1997).

Numerous other factors have been reported to be associated with a person's likelihood of complying with harm command hallucinations including: accepting the voice as real (Erkwoh, Willmes, Eming-Erdmann, \& Kunert, 2002), having delusions that fit with what the command hallucinations is ordering (Junginer, 1990), a history of prior suicide attempts in response to harm command hallucinations (Harkavy-Friedman, Kimhy, Nelson, Venarde, Malaspina, \& 
Mann, 2003), having low self-control over the voice (Beck-Sander, et al., 1997), viewing the voice as intrusive, and having fewer coping strategies (Mackinnon et al., 2004).

In summary, harm command hallucinations are prevalent and of serious social concern as they can be associated with suicide and violence towards others. The characteristics of people who hear harm command hallucinations and whether a course that teaches behavioral management strategies for coping with voices is effective with command hallucinations has not been reported.

\section{Method}

This study used a repeated measures design to assess auditory hallucinations, depression and anxiety at baseline (before the course), end of course, and one-year post course. The specific protocol, methodology and sample are described in detail elsewhere (Buccheri et al., 2004; Trygstad et al. 2002). Approval from institutional review boards and informed consent from participants were obtained.

\section{Measurement and Instrumentation}

Auditory Hallucinations Interview Guide-Long Form (AHIG-LF). This 50-item interview guide developed by the authors asks for demographic and detailed information about auditory hallucinations, substance use, and psychiatric medications (Buccheri et al., 1996).

\section{Characteristics of Auditory Hallucinations Questionnaire-Expanded Version (CAHQ-EV).}

The expanded version of the CAHQ included two additional questions related to hearing command hallucinations to harm self and/or others. The CAHQ is a 7-item Likert-type instrument on which participants rate characteristics of their auditory hallucinations (i.e., frequency, loudness, self-control, clarity, tone, distractibility and distress) over the past 24 hours 
on a scale from 1-5. Higher scores indicate more negative characteristics (e.g., more frequent, louder, less self-control) (Buccheri, et al., 2004; Trygstad et al., 2002).

Helpfulness of course. For this study, 2 Likert-type items were developed to assess expected and actual perceived helpfulness of the course. Responses on each item ranged from 1 "not at all helpful" to 5 "extremely helpful”.

Unpleasant Voices Scale (UVS). This 5-item scale, developed by the authors, asks participants to rate the intensity of their unpleasant voices on a scale of 0 "no voices heard" to 10 "the most unpleasant your voices could be" during the past 24 hours and past week.

Tension-anxiety subscale of the Profile of Mood States (POMS). This is a 9-item subscale that is measured on a 5-point Likert scale, ranging from 0 "not at all" to 4 "extremely". A higher score indicates a higher level of anxiety (McNair, Lorr, \& Droppleman, 1992).

Beck Depression Inventory, second edition (BDI-II). This 21-item self-report instrument assesses depressive symptoms, with higher scores indicating a higher level of depression (0-13 minimal, 14-19 mild, 20-28 moderate and 29-63 severe) (Beck, Steer, \& Brown, 1996).

\section{Data Analyses and Results}

The instruments were completed by 57 participants at baseline, 50 at end of course, and 46 at one-year post course.

Prevalence. At baseline, $47 \%(\mathrm{n}=27)$ of participants reported hearing at least one type of harm command hallucination. Forty-four percent reported hearing commands to harm self and $21 \%$ to harm others. There was overlap between the two categories, $16 \%$ reported hearing both command hallucinations to harm self and to harm others. Seventy-five percent of participants who heard commands to harm others also heard commands to harm self while only $36 \%$ of those who heard commands to harm self also reported hearing commands to harm others. 
Demographic and clinical characteristics. Medians were calculated for continuous variables due to the skewed nature of the data. The median age of participants hearing harm commands at baseline was 44 years, participants first heard voices at a median age of 22 , and the median number of voices heard was 4 . Frequencies were calculated for all other demographic and clinical characteristics. Review of demographic characteristics at baseline revealed more women (71\%) than men $(38 \%)$ heard harm command hallucinations.

At baseline, $100 \%$ of participants who heard harm command hallucinations reported that their voices were critical of them, $81 \%$ reported that their voices commented on what they were doing, $59 \%$ heard sounds other than voices, $56 \%$ heard music, $48 \%$ were awakened at night by their voices and $30 \%$ reported that "during the night" was their worse time in a 24 -hour period. These findings and others are presented in Table 1.

Changes in: a) prevalence; b) characteristics and intensity of auditory hallucinations; and c) levels of anxiety and depression immediately after attending the course and at one-year post course. Immediately after attending the course, the prevalence of command hallucinations to harm self decreased from $44 \%$ to $24 \%$ and remained at $24 \%$ one-year post course. The prevalence for command hallucinations to harm others decreased from $21 \%$ to $16 \%$ immediately after attending the course and was $17 \%$ one-year post course. Mean scale scores at the three measurement time points are presented in Figures 1 and 2.

Perceived helpfulness of the course. Mean scores for participants' baseline expected helpfulness $(\mathrm{M}=3.63 ; \mathrm{SD} \pm 0.90)$, actual helpfulness post course $(\mathrm{M}=3.81 ; \mathrm{SD} \pm 0.87)$ and actual helpfulness at one-year post course $(M=3.70 ; S D= \pm 0.87)$ were all between 3 "moderately helpful" and 4 "very helpful". The course was more helpful than participants expected and most helpful immediately post course. 
Effectiveness of the 10 strategies taught in the course. Participants were asked at the end of the course which strategies worked best for them when they heard command hallucinations to harm self and/or others. Each participant reported having their own unique "toolbox" of strategies that they could use when command hallucinations were bothering them. All 10 strategies that were taught in the course were being used by someone and some participants had incorporated two additional strategies not taught in the course, prayer and calling for professional help. The 10 strategies taught were used regardless of whether participants heard command hallucinations to harm self, harm others or both. No one particular strategy worked best for everyone with harm command hallucinations or with a particular type of command hallucinations.

How individual data differed from grouped data. Individual analyses provided us with information not available from analysis of grouped data about the different patterns participants experienced with hearing command hallucinations over time.

Command hallucinations to harm self were found to be persistent. Participants reported having them "from birth", from "childhood" and for "years on end." The course was effective in reducing the number of participants who heard commands to harm self with the percentage decreasing from $52 \%$ at baseline to the end of the course, and by $56 \%$ from baseline to one-year post course. At the one-year follow-up, one participant who had not reported hearing command hallucinations to harm self at baseline, reported hearing them. These findings are displayed in Table 2 .

The participants who heard command hallucinations to harm others reflected more variability/change at each data collection point than those with command hallucinations to harm self. The course was even more effective in reducing the number of participants who heard 
commands to harm others than harm self. The number of participants who heard command hallucinations to harm others at baseline decreased by $58 \%$ at the end of the course and $83 \%$ from baseline to one-year post course. However, three participants who had not heard command hallucinations to harm others at baseline reported hearing them at the end of the course and four participants who had not reported hearing them at baseline or at end course reported hearing them at one-year post course. Neither the improvement in those who heard command hallucinations to harm others at baseline $(n=12)$ and no longer heard them at the end of the course $(n=7)$ or at one-year post course $(n=10)$, nor the number of new hearers of command hallucinations to harm others at the end of the course $(n=3)$ or at one-year post course $(n=4)$ were evident in the grouped data.

Command hallucinations to harm others appear to be more variable than command hallucinations to harm self. For some participants with both commands to harm self and others, commands to harm others went away and came back but commands to harm self remained constant.

Individual analysis of demographic and clinical characteristics. We wondered if there were demographic or clinical characteristics of study participants that might predict those whose harm command hallucinations would stop after completing the course. To this end, we identified a group whose harm command hallucinations stopped by the end of the course and were still absent at one-year post course, and a second group who consistently heard harm command hallucinations at all three data points. A third group emerged from the data whose harm command hallucinations were present at some data collection points but not all. Results are presented in Table 3 . 


\section{Discussion}

Nearly half (47\%) of the participants in this study reported hearing harm command hallucinations. This was similar to the median (48\%) and within the range (7-70\%) of those with command hallucinations who hear commands telling them to do harmful or dangerous acts reported by Shawyer et al. (2003).

Hearing command hallucinations to harm self was associated with hearing command hallucinations to harm others and vice versa. For patients who have harm command hallucinations, clinicians should assess whether they are hearing commands to harm self, others or both.

The overall prevalence of both types of harm command hallucinations were reduced after attending the course. In addition, all seven characteristics (i.e., frequency, loudness, self-control, clarity, tone, distractibility, distress) and intensity of auditory hallucinations, anxiety and depression showed improvement after attending the course with even more improvement seen at one-year post course.

Female participants were more likely than males to hear harm command hallucinations. Females were also more likely to hear commands to harm self that were persistent, while males were more likely to hear commands to harm self that came and went. Further exploration into how men and women differ in hearing harm command hallucinations and examining other potential relationships among a variety of demographic variables is needed.

Participants who heard harm command hallucinations reported being frequently distressed by their voices at night. A number of participants in the study were in living situations where their stay was jeopardized if they woke up other residents, staff or family members during the night. Clients reported fearing that they might disturb others if they used strategies such as listening to 
music, watching TV, talking to someone or calling Suicide Prevention or a crisis unit. In some settings, there was limited access to medication during the night. More research is needed to further identify effective management strategies and resources for people who hear command hallucinations at night. For example, is having a CD player with headphones next to the bed or having a PRN dose of an antipsychotic or anti-anxiety medication available helpful for those who are awakened at night and distressed by their command hallucinations.

Command hallucinations to harm others were less stable than command hallucinations to harm self and were reported to go away and come back and seldom lasted for as long as a year. The frequency of command hallucinations to harm self and others and the more constant nature of command hallucinations to harm self in comparison to the fluidity of command hallucinations to harm others need further research.

Alcohol and/or drug use may be a factor that contributes to the variability of harm command hallucinations. In analyzing individual data for those whose harm command hallucinations stopped or continued after attending the course, all reported being either "clean and sober" or "minimal or decreasing" substance use. However, for the group who reported their harm command hallucinations as variable, $44 \%$ percent reported "regular" use of alcohol or drugs and one reported "regular" use of alcohol as a coping strategy.

The high prevalence of harm command hallucinations in our sample led us to add specific questions to the Unpleasant Voices Scale about whether participants hear harm command hallucinations and to call for the development of a Protocol Response to Suicidal Ideation and Homicidal Ideation (Gerlock, 2006). This is a written protocol that goes with the Unpleasant Voices Scale to be used if a participant answers yes to questions about whether they are hearing harm command hallucinations. 
The overall helpfulness of the course was clear from our results, however no one "best" strategy was consistently identified. This is similar to our findings for persistent auditory hallucinations as a whole (Buccheri et al. 1996, 1997, 2004; Trygstad et al. 2002). Therefore, patients with harm command hallucinations should be advised to try all 10 strategies and to identify the ones that work best for them.

\section{Conclusion}

Command hallucinations to harm self and others are prevalent and often occur together. The prevalence of both command hallucinations to harm self and others decreased after attending the course and remained decreased one-year post course. People with harm command hallucinations who took our course perceived it as helpful and, at the end of the course and one-year post course, demonstrated improvement in all seven of the characteristics and intensity of auditory hallucinations, and levels of anxiety and depression. All strategies were useful to some participants and no one strategy worked best for everyone. Individual data analysis added information beyond what was available from grouped data. Individual data analysis, and analysis of demographic and clinical characteristics of those who heard command hallucinations to harm self or others provided directions for further research.

\section{Current Status}

This Behavioral Management of Auditory Hallucinations Treatment Program is currently being implemented in national and international sites as a dissemination project with a program evaluation component. If you would like to teach this course in your setting, please contact Robin Buccheri at the following e-mail address: managingvoices@usfca.edu. 


\section{References}

Associated Press (AP). (2005, October 21). Mentally ill mom pleads innocent to murder of 3 sons. USA Today. Retrieved online at www.usatoday.com on June 23, 2006.

Beck, A. T., Steer, R. A., \& Brown, G. K. (1996). BDI-II manual. San Antonio, TX: Harcourt Brace.

Beck-Sander, A., Birchwood, M., \& Chadwick, P. (1997). Acting on command hallucinations: A cognitive approach. British Journal of Clinical Psychology, 36, 139-148.

Buccheri, R., Trygstad, L., Dowling, G., Hopkins, R., White, K., Griffin, J., et al. (2004). Long term effects of teaching behavioral strategies for managing persistent auditory hallucinations in schizophrenia. Journal of Psychosocial Nursing and Mental Health Services, 42, 18-27.

Buccheri, R. K., Trygstad, L., Kanas, N., \& Dowling, G. (1997). Symptom management of auditory hallucinations in schizophrenia: Results of 1-year follow up. Journal of Psychosocial Nursing and Mental Health Services, 35, 20-28.

Buccheri, R. K., Trygstad, L., Kanas, N., Waldron, B., \& Dowling, G. (1996). Auditory hallucinations in schizophrenia: Group experience in examining symptom management and behavioral strategies. Journal of Psychosocial Nursing and Mental Health Services, 34, 12-25.

Erkwoh, R., Willmes, K., Erming-Erdmann, A., \& Kunert, H. J. (2002). Command hallucinations: Who obeys and who resists when? Psychopathology, 35, 272-279.

Gerlock, A. (2006). Protocol response to Suicidal Ideation/Homicidal Ideation. Unpublished manuscript.

Harkavy-Friedman, J. M., Kimhy, D., Nelson, E. A., Venarde, D. F., Malaspina, D., \& Mann, J.J. (2003). Suicide attempts in schizophrenia: The role of command auditory hallucinations for suicide. Journal of Clinical Psychiatry, 64(8), 871-874. 
Junginger, J. (1990). Predicting compliance with command hallucinations. American Journal of Psychiatry, 147, 245-247

Junginger, J. (1995). Command hallucinations and the prediction of dangerousness. Psychiatric Services, 46(9), 911-913.

Kasper, M. E., Rogers, R., \& Adams, P. A. (1996). Dangerousness and command hallucinations: An investigation of psychotic inpatients. Bulletin of the American Academy of Psychiatry and the Law, 24(2), 219-224.

Mackinnon, A., Copolov, L., \& Trauer, T. (2004). Factors associated with compliance and resistance to command hallucinations. The Journal of Nervous and Mental Disease, 192(5), 357362.

McNair, D. M., Lorr, M., \& Droppleman, L. F. (1972). Edits manual for the Profile of Mood States. San Diego, CA: EDITS/Educational and Industrial Testing Service.

Rudnick, A. (1999). Relation between command hallucinations and dangerous hallucinations and dangerous behavior. Journal of American Academy of Psychiatry Law, 27(2), 253-257.

Shawyer, F., Mackinnon, A., Farhall, J., Trauer, T., \& Copolov, D. (2003). Command hallucinations and violence: Implications for detention and treatment. Psychiatry, Psychology and Law, 10(1), 97-107.

Trygstad, L., Buccheri, R., Dowling, G., Zind, R., White, K., Griffin, J., et al. (2002). Behavioral management of persistent auditory hallucinations in schizophrenia: Outcomes from a 10-week course. Journal of the American Psychiatric Nurses Association, 8(3) 84-91. 
Table 1

Baseline Clinical Characteristics of Participants with Harm Command Hallucinations

\begin{tabular}{|l|l|}
\hline Clinical Characteristics & Percent \\
\hline & \\
\hline Know the Speaker & 48 \\
\hline Person from the Past & 33 \\
\hline Voices are Continuous Monologue & 44 \\
\hline Voices Last One Hour or Longer & 41 \\
\hline Voices Comment on What They are Doing & 81 \\
\hline Voices are Critical of Them & 100 \\
\hline Voices Laugh at Them & 54 \\
\hline Voices Have Religious Theme & 50 \\
\hline Voices Have Sexual Theme & 46 \\
\hline Voices Talk to Them & 93 \\
\hline Voices Talk to Each Other & 48 \\
\hline Hear Music & 56 \\
\hline $\begin{array}{l}\text { Hear Other Sounds (e.g., door opening and } \\
\text { closing, muffled scream) }\end{array}$ & 59 \\
\hline Awakened at Night By Voices & 48 \\
\hline $\begin{array}{l}\text { During the Night is Worst Time in a 24- } \\
\text { Hour Period }\end{array}$ & 30 \\
\hline
\end{tabular}




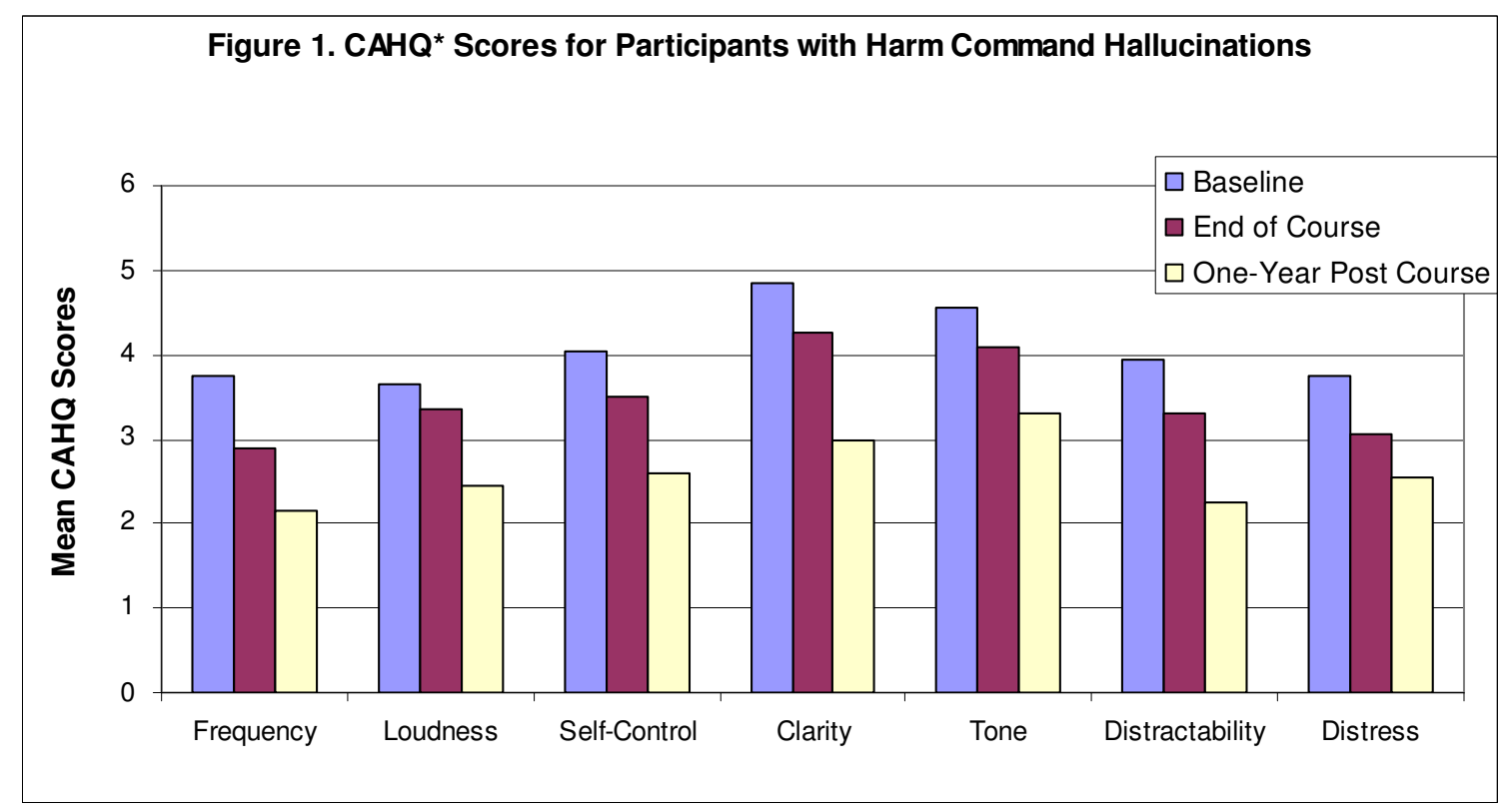

${ }^{*}$ Characteristics of Auditory Hallucinations Questionnaire

Figure 2. UVS ${ }^{\star}$, POMS $^{\star \star}$, and BDI-II ${ }^{\star \star \star}$ Scores for Participants with Harm Command Hallucinations

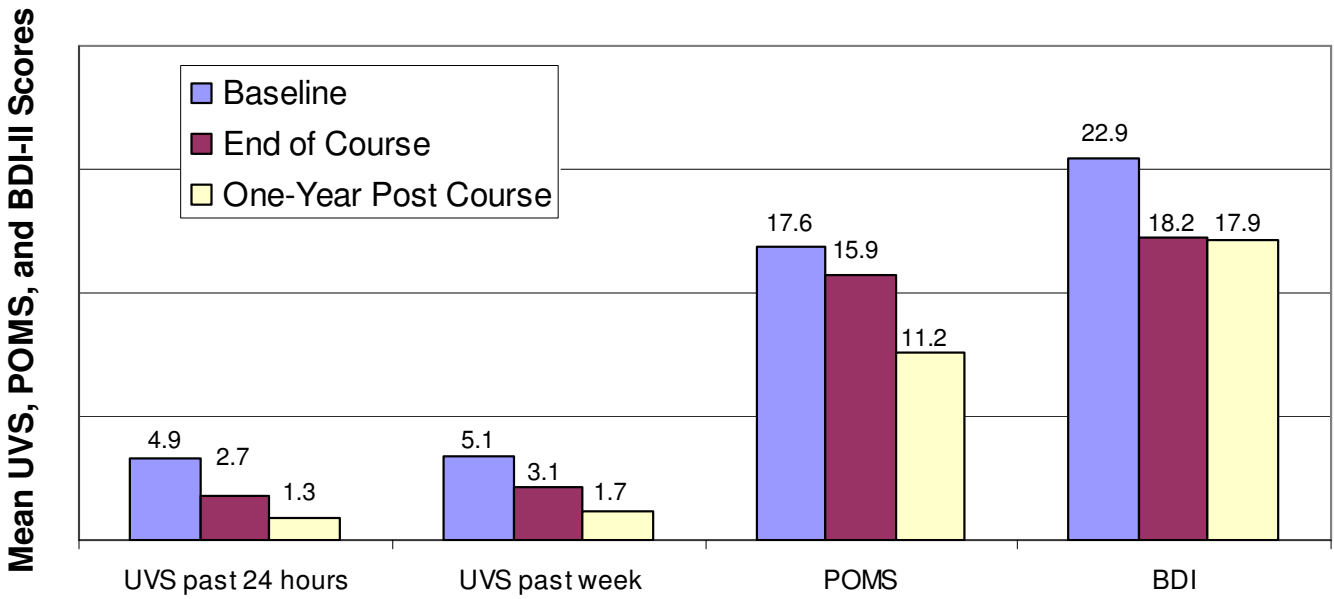

* Unpleasant Voices Scale

** Profile of Mood States - Tension - Anxiety Subscale

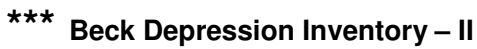


Table 2

Analysis of Individual Data:

Command Hallucinations to Harm Self and Others

\begin{tabular}{|c|c|c|c|}
\hline & Baseline & End of Course & One Year Post-Course \\
\hline Harm self & $\mathrm{n}=25$ & $\frac{\mathrm{n}=12 \text { total }}{12 \text { heard at baseline }}$ & $\begin{array}{l}\mathrm{n}=11 \text { total } \\
10 \text { heard at baseline } \\
1 \mathrm{new}\end{array}$ \\
\hline Harm others & $\mathrm{n}=12$ & $\begin{array}{l}\frac{\mathrm{n}=8 \text { total }}{5 \text { heard at baseline }} \\
3 \text { new }\end{array}$ & $\begin{array}{l}\mathrm{n}=8 \text { total } \\
2 \text { heard at baseline } \\
2 \text { heard at end of } \\
\text { course } \\
4 \text { new }\end{array}$ \\
\hline
\end{tabular}


Table 3

Demographic and Clinical Characteristics of Participants with Harm CH

\begin{tabular}{|c|c|c|c|}
\hline Characteristic & $\begin{array}{c}\text { Group 1: Baseline } \\
\text { Command } \\
\text { Hallucinations were } \\
\text { Absent Both at End of } \\
\text { Course and One-Year } \\
\text { Post Course } \\
(n=8)\end{array}$ & $\begin{array}{c}\text { Group 2: Baseline } \\
\text { Command } \\
\text { Hallucinations } \\
\text { Continued at Both } \\
\text { End of Course and } \\
\text { One-year Post Course } \\
(n=5)\end{array}$ & $\begin{array}{c}\text { Group } 3 \text { Command } \\
\text { Hallucinations were } \\
\text { Variable from } \\
\text { Baseline to End of } \\
\text { Course to One-Year } \\
\text { Follow-up } \\
(n=9)\end{array}$ \\
\hline \multicolumn{4}{|l|}{ Sex } \\
\hline Male & $63 \%$ & $20 \%$ & $78 \%$ \\
\hline Female & $37 \%$ & $80 \%$ & $22 \%$ \\
\hline \multicolumn{4}{|l|}{ Ethnicity } \\
\hline Caucasian & $37 \%$ & $60 \%$ & $89 \%$ \\
\hline African American & $25 \%$ & 0 & 0 \\
\hline Latino & $13 \%$ & 0 & $11 \%$ \\
\hline Chinese & $13 \%$ & $40 \%$ & 0 \\
\hline Other & $13 \%$ & 0 & 0 \\
\hline \multicolumn{4}{|l|}{$\begin{array}{l}\text { Started Hearing } \\
\text { Voices as a Child }\end{array}$} \\
\hline Yes & 0 & $60 \%$ & $11 \%$ \\
\hline $\mathrm{No}$ & $100 \%$ & $40 \%$ & $89 \%$ \\
\hline \multicolumn{4}{|l|}{$\begin{array}{l}\text { Voices Have Gotten } \\
\text { Better at Some Point } \\
\text { in the Past }\end{array}$} \\
\hline Yes & $63 \%$ & $40 \%$ & $11 \%$ \\
\hline No & $37 \%$ & $60 \%$ & $89 \%$ \\
\hline \multicolumn{4}{|l|}{$\begin{array}{l}\text { Number of Voices } \\
\text { Heard }\end{array}$} \\
\hline 1 & $43 \%$ & $20 \%$ & 0 \\
\hline $2-5$ & $29 \%$ & $40 \%$ & $78 \%$ \\
\hline 6 or more & $29 \%$ & $40 \%$ & $22 \%$ \\
\hline $\begin{array}{l}\text { Laughed at by Voices } \\
\text { Yes }\end{array}$ & $13 \%$ & $80 \%$ & $33 \%$ \\
\hline No & $87 \%$ & $20 \%$ & $67 \%$ \\
\hline $\begin{array}{l}\text { Voices Critical } \\
\text { Yes }\end{array}$ & $100 \%$ & $100 \%$ & $89 \%$ \\
\hline No & 0 & 0 & $11 \%$ \\
\hline \multicolumn{4}{|l|}{ Regular Alcohol Use } \\
\hline Yes & 0 & 0 & $44 \%$ \\
\hline
\end{tabular}




\begin{tabular}{|l|l|l|l|}
\hline No & $100 \%$ & $100 \%$ & $66 \%$ \\
\hline $\begin{array}{l}\text { *BDI-II: moderate } \\
\text { score at baseline-not } \\
\text { sure this is } \\
\text { accurate???? Did all } \\
\text { scores fall between 20 } \\
\text { and 28??? Or were } \\
\text { there some higher } \\
\text { than 28 which would } \\
\text { be severe if so we } \\
\text { could say "moderate } \\
\text { to severe score at } \\
\text { baseline" }\end{array}$ & & & \\
\hline Yes & & & \\
\hline No & & & \\
\hline $\begin{array}{l}\text { On Mood Altering } \\
\text { Drugs (i.e., mood } \\
\text { stabilizers, } \\
\text { antidepressants and/or } \\
\text { antianxiety agents) }\end{array}$ & & & \\
\hline Yes & & & \\
\hline No & & & \\
\hline
\end{tabular}

*Beck Depression Inventory-II 